\title{
Reoperative surgery on the thoracoabdominal aorta
}

\author{
Joseph S. Coselli, MD, ${ }^{\mathrm{a}, \mathrm{b}, \mathrm{c}, \mathrm{d}}$ Cristian Rosu, MD, ${ }^{\mathrm{a}, \mathrm{c}, \mathrm{d}}$ Hiruni S. Amarasekara, MS,, a,c,ed \\ Susan Y. Green, MPH, ${ }^{\mathrm{a}, \mathrm{c}, \mathrm{d}, \mathrm{e}}$ Qianzi Zhang, MPH, ${ }^{\mathrm{e}}$ Matt D. Price, MS, ${ }^{\mathrm{a}, \mathrm{d}, \mathrm{d}, \mathrm{e}}$ and Scott A. LeMaire, MD ${ }^{\mathrm{a}, \mathrm{b}, \mathrm{c}, \mathrm{d}, \mathrm{e}}$
}

\section{ABSTRACT}

Objective: Since the advent of endovascular repair for aortic aneurysms, many centers have justified the use of endovascular approaches in patients with previous open distal aortic repair by deeming these patients "high risk" because of their previous operation. We sought to determine whether patients who undergo reoperative repair for thoracoabdominal aortic aneurysm (TAAA) have worse outcomes than patients who undergo non-reoperative repair.

Methods: We reviewed our data on 3379 TAAA repairs from 1986 to 2016. We compared patients' preoperative characteristics, surgical variables, and outcomes among reoperative $(n=726)$ and non-reoperative $(n=2653)$ cases. Furthermore, we examined reoperative indications to identify repairs performed because of repair failure $(\mathrm{n}=93)$ and reoperations performed as an adjacent extension of repair $(\mathrm{n}=633)$. A multivariable analysis was conducted to identify predictors of adverse events by using relevant preoperative and intraoperative factors.

Results: The operative mortality rate did not significantly differ between groups $(8.1 \%$ for reoperative vs $7.3 \%$ for non-reoperative; $P=.5)$; in addition, reoperative repair was not associated with an increased risk of adverse event. However, Kaplan-Meier survival analysis showed that over the first 10 years, the reoperative groups fared significantly worse than the non-reoperative group $(P<.001)$ (survival estimates at 10 years: $23.9 \% \pm 4.9 \%$ for patients with repair failure, $28.4 \% \pm 2.0 \%$ for those with extension of repair, and $40.1 \% \pm 1.1 \%$ for non-reoperative repairs).

Conclusions: We were unable to detect noteworthy differences in early outcomes between reoperative and non-reoperative TAAA repair. However, mid-term results indicate worse survival for patients who undergo reoperative surgery. (J Thorac Cardiovasc Surg 2018;155:474-85)

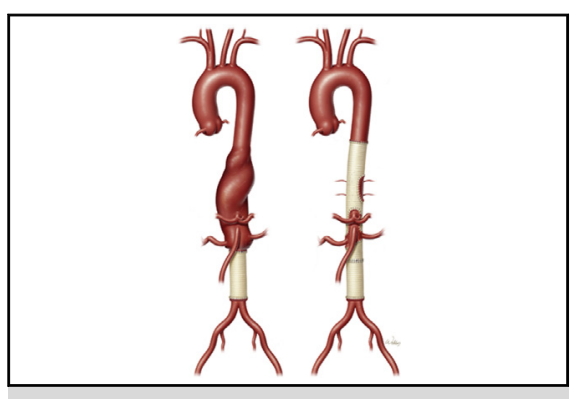

Illustrations demonstrate a common form of reoperative thoracoabdominal aortic aneurysm repair.

\section{Central Message}

Reoperative TAAA repair yields respectable early outcomes that do not significantly differ from those of non-reoperative TAAA repair.

\section{Perspective}

Reoperative thoracoabdominal aortic surgery is typically necessitated by (1) complications of a previous open repair or (2) aortic disease that progresses into an aortic segment adjacent to an intact previous open repair. Although reoperative surgery may necessitate a modified approach, such repair produces respectable early outcomes that do not significantly differ from those of non-reoperative TAAA repair.

See Editorial Commentary page 486.

See Editorial page 459.
Reoperative surgery on the thoracoabdominal aorta is not uncommon. In our recent report of 3309 thoracoabdominal aortic aneurysm (TAAA) repairs, ${ }^{1}$ more than one quarter of

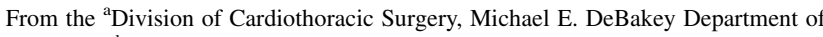
Surgery, ${ }^{\mathrm{b}}$ Cardiovascular Research Institute, and ${ }^{\mathrm{e}}$ Surgical Research Core, Michael E. DeBakey Department of Surgery, Baylor College of Medicine; ${ }^{\mathrm{c}}$ Adult Cardiac Service, CHI St. Luke's Health-Baylor St. Luke's Medical Center, and ${ }^{\mathrm{d}}$ Department of Cardiovascular Surgery, Texas Heart Institute, Houston, Tex.

Read at The American Association for Thoracic Surgery Aortic Symposium, New York, New York, May 12-13, 2016.

Received for publication Aug 30, 2016; revisions received July 18, 2017; accepted for publication Aug 9, 2017; available ahead of print Sept 22, 2017.

Address for reprints: Scott A. LeMaire, MD, One Baylor Plaza, BCM 390, Houston, TX 77030 (E-mail: slemaire@bcm.edu).

$0022-5223 / \$ 36.00$

Copyright (c) 2017 by The American Association for Thoracic Surgery

http://dx.doi.org/10.1016/j.jtcvs.2017.08.024
}

the repairs $(\mathrm{n}=858 ; 25.9 \%)$ involved patients who had undergone a previous repair of the distal aorta (descending thoracic, thoracoabdominal, or abdominal aorta). Other aortic centers have reported $13 \%$ to $22 \%$ rates of previous distal aortic repair in patients undergoing TAAA repair, with the bulk of previous repairs involving the infrarenal abdominal aorta. $^{2-6}$

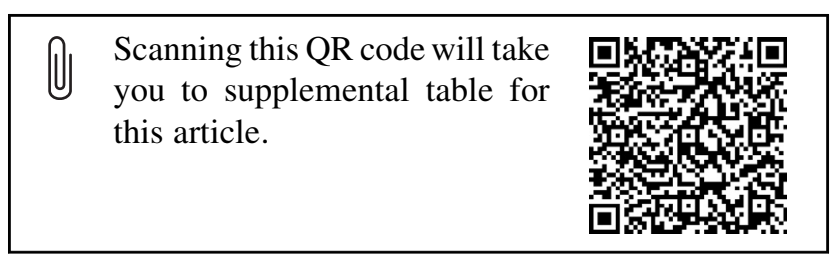




\section{Abbreviations and Acronyms}

AAA $=$ abdominal aortic aneurysm

CSF $=$ cerebrospinal fluid

DTA $=$ descending thoracic aneurysm

TAAA $=$ thoracoabdominal aortic aneurysm

Since the advent of endovascular repair for aortic aneurysms, many centers have justified the use of endovascular approaches in patients with previous open aortic repair by deeming these patients "high risk" because of their previous operation. ${ }^{7}$ Concerns about enhanced risk are supported by the wellunderstood risk of bleeding and other complications during re-entry of potentially hostile thoracic and abdominal cavities, as well as by evidence suggesting elevated rates of paraplegia after TAAA repair in patients with previous open abdominal aortic aneurysm (AAA) repair. ${ }^{8}$ Conversely, other reports ${ }^{9,10}$ suggest that the risk of postoperative paraplegia can be reduced by "staging" open distal repairs to permit the development of a collateral network of arteries that can improve spinal perfusion between repairs. Despite considerable interest in these issues, the literature contains few reports that specifically describe TAAA repair after previous open descending thoracic aneurysm (DTA), TAAA, or AAA repair or that describe the risks associated with "redo" TAAA repair ${ }^{4,5,11-15}$; thus, we sought to better assess the risk of paraplegia and other complications after reoperative TAAA repair, both in comparison with nonreoperative repair and by indication for reoperation.

\section{PATIENTS AND METHODS}

\section{Study Enrollment and Patient Characteristics}

Baylor College of Medicine's institutional review board approved our clinical research protocol in 2006. For patients who underwent surgery after protocol approval, data were collected prospectively, and informed consent was obtained whenever possible; waiver of consent was approved for patients who could not provide consent because of illness and whose family members were not available. For patients who underwent surgery before protocol approval, data were collected retrospectively from medical records, and consent was waived.

We examined our experience involving 3379 TAAA repairs to identify factors associated with reoperation and to determine whether risk is greater in reoperative TAAA surgery than in non-reoperative surgery. We theorized that reoperative TAAA surgery is typically necessitated by 1 of 2 factors: (1) complications signaling the failure of a previous open repair (Figure 1) or (2) aortic disease that progresses into an aortic segment adjacent to an intact previous open repair (Figure 2).

Of 3379 TAAA repairs performed between 1986 and 2016, 726 repairs $(21.5 \%)$ were identified as reoperative and the other 2653 repairs $(78.5 \%)$ were considered non-reoperative (Figure 3). Indications for reoperation were characterized as TAAA repair failure $(\mathrm{n}=93 ; 2.8 \%)$ or progression of disease necessitating extension of repair to an adjacent aortic segment $(\mathrm{n}=633 ; 18.7 \%)$. To better elucidate the characteristics of patients in

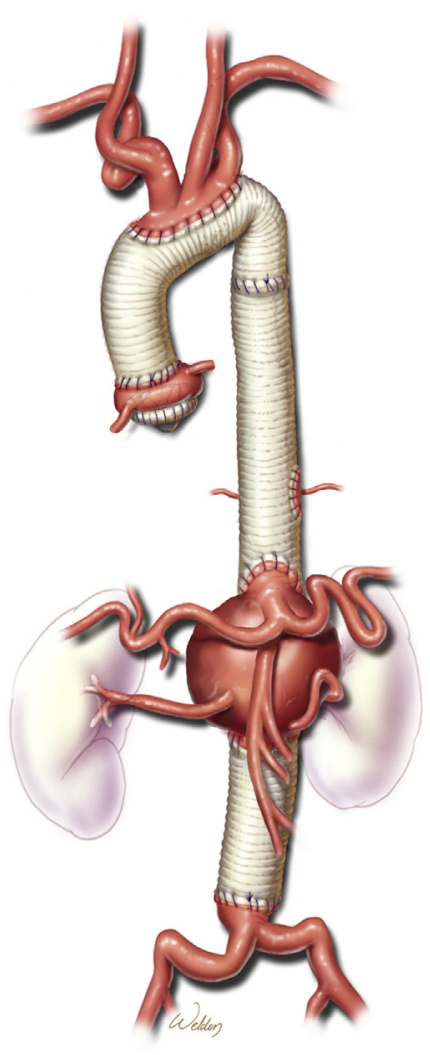

FIGURE 1. Drawing illustrates late repair failure of open TAAA surgery. In the residual native aortic tissue of the visceral patch, an aneurysm has formed.

need of reoperative surgery after open repair, we did not include in the reoperative group patients who underwent operations performed after entirely endovascular aortic repairs, second-stage operations for completion of elephant trunk repair (if no other distal aortic repair had been performed previously; $\mathrm{n}=140$ ), or operations in which the previous repair involved a noncontiguous portion of the distal aorta (such as an extent I TAAA repair after a previous infrarenal AAA repair; $n=110$ ) (Table 1). These repairs remained in the overall series but were categorized as non-reoperative TAAA repairs.

Of the 93 repair failures, few involved graft infection $(n=5)$, graft-related fistula $(\mathrm{n}=1)$, graft rupture $(\mathrm{n}=2)$, or expansion after aortoplasty $(\mathrm{n}=1)$. Most repair failures manifested as pseudoaneurysm $(\mathrm{n}=44)$, patch aneurysm $(\mathrm{n}=30)$, or both $(\mathrm{n}=10)$. In the extension-of-repair group $(n=633)$, the majority of the previous distal open aortic repairs were infrarenal AAA repairs $(436 / 633 ; 68.9 \%)$ (Table 1), and most of these were performed outside our center (572/633; $90.4 \%)$. In addition, $10.1 \%(\mathrm{n}=75)$ of the 726 reoperative repairs were made in patients with more than 1 previous distal open aortic repair; this proportion was $25.8 \%$ in the repair-failure group (ie, 24 of 93 patients had more than 1 previous distal aortic repair).

\section{Study Definitions and Follow-up}

As reported in recent publications, all preoperative, operative, and outcomes data were collected with the use of standard definitions. ${ }^{1,16}$ Reoperative surgery was defined as that necessitated by repair failure or that involved extension of a contiguous repair; any procedure that did not meet this criterion was considered non-reoperative. We defined repair failure as any complication of previous open distal aortic repair that necessitated aortic reoperation: pseudoaneurysm, patch aneurysm, 

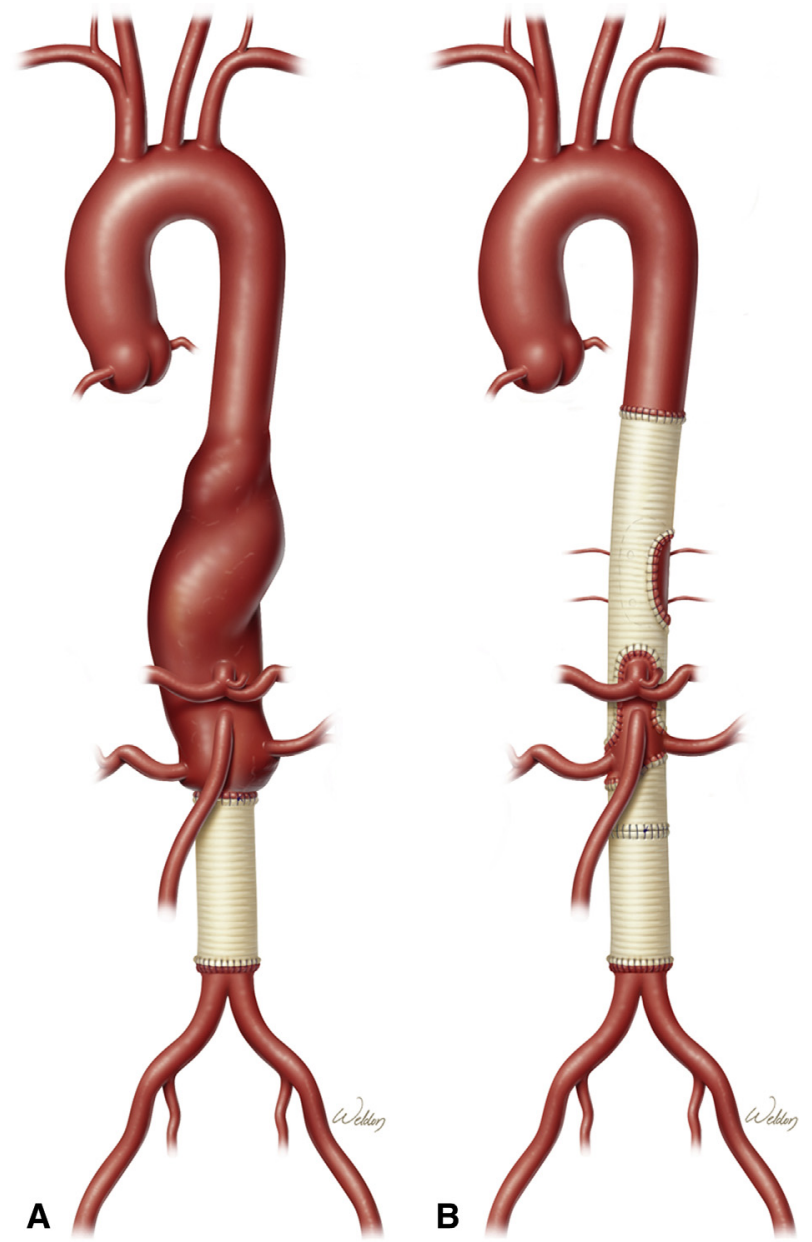

FIGURE 2. Drawings illustrate a common scenario involving reoperative TAAA surgery. A, An extent III TAAA has developed above the intact previous open extent III TAAA repair. B, To treat the progression of aneurysmal disease, open repair was performed. The previous graft is incorporated into the distal anastomosis.

expansion after aortoplasty, graft infection, graft rupture, or graft-related fistula. We defined extension of repair as repair necessitated by the progression of disease into aortic segments that are adjacent to and contiguous with the previously repaired segment; in most cases, the previously placed graft was left in place.

Patients were considered symptomatic if they had symptoms indicative of aortic disease (eg, pain, dysphagia, hoarseness). To estimate the glomerular filtration rate, we used the Chronic Kidney Disease Epidemiology Collaboration equation. ${ }^{17}$ Repairs were divided into 3 eras based on the adoption and use of key surgical adjuncts: Era 1 (1986-1998; before the use of key adjuncts became standard practice; $\mathrm{n}=1158)$, Era 2 (1999-2004; early adoption of key adjuncts; $\mathrm{n}=1059$ ), and Era 3 (2005-2016; contemporary use of key adjuncts; $n=1162){ }^{1,18}$ Operative death was defined as death that occurred within 30 days of surgery or during the index hospitalization (ie, before final discharge from our hospital or from any other hospital or long-term acute care facility to which the patient was transferred postoperatively). Adverse event, a composite endpoint, was defined as operative death or discharge with any of the following complications: renal failure necessitating dialysis, paraplegia, paraparesis, or stroke. ${ }^{19}$ Complications were considered persistent if they were present at the time of early death or hospital discharge. Early survivors were those patients who survived to hospital discharge. Clinical follow-up information was obtained by clinic visit, telephone interview, or written correspondence. The Social Security Death Index was used to identify deaths among patients who were lost to follow-up.

\section{Surgical Techniques}

Our technique for open TAAA repair has been refined over time, follows a multimodal strategy that is largely based on the Crawford classification scheme for defining the extent of TAAA repair, and generally reserves the use of surgical adjuncts for extensive aortic repair (extents I and II). Our 30-year clinical practice is described in greater detail elsewhere, and for the last decade, our approach largely has been standardized. ${ }^{1,18}$ In brief, we routinely use moderate systemic heparinization $(1.0 \mathrm{mg} / \mathrm{kg})$, permit mild passive hypothermia $\left(32^{\circ} \mathrm{C}-34^{\circ} \mathrm{C}\right)$, use cold renal perfusion $\left(4^{\circ} \mathrm{C}\right)$ whenever the renal ostia are accessible, and, when appropriate, reattach at least 1 pair of intercostal or lumbar arteries. For extensive aortic repair, we typically use left heart bypass, selective visceral perfusion, and cerebrospinal fluid (CSF) drainage; in less-extensive repairs (extents III and IV), these adjuncts are not used commonly but may be selectively added, such as in cases of reoperation after previous abdominal aortic replacement or endovascular aneurysm repair. As needed, we treat the 4 visceral arteries (the celiac axis, superior mesenteric artery, and both renal arteries) with bypass grafts, endarterectomy, or stents.

Whereas our overall strategy is similar for reoperative and non-reoperative TAAA repair, we modify our approach for reoperations with an awareness that the need to lyse adhesions adds complexity to the procedure and unavoidably lengthens its overall duration. Further modifications for reoperative surgery include efforts to make an offset incision through a different intercostal space and to limit exposure whenever feasible so as to avoid bleeding complications and unintentional injury of nearby structures when adhesions are dissected. Sharp dissection is typically used to free adhesions that hamper exposure, but an electrocautery may be selectively used at low power settings. In addition, we tend to minimize lung retraction during anticoagulation so as to minimize the risk of bleeding. If separating the lung from the chest wall results in parenchymal injury, we often apply a sealant to the involved area of the lung before closing the chest. Because adhesions involving the spleen are common, we carefully inspect the spleen before closure. If a small capsular tear or other damage to the spleen is found, it may be possible to repair it by using cautery and Surgicel (Ethicon US, LLC, Cincinnati, Ohio); however, if the tear cannot be repaired, the spleen generally is removed. In our previous report on reoperative surgery, ${ }^{20}$ we noted that the aggressive use of blood products appeared to minimize bleeding complications and that fresh-frozen plasma and platelets particularly are helpful in ameliorating perioperative bleeding. In addition, in reoperative surgery, we use CSF drainage more liberally; for example, we often use CSF drainage during extent III and IV repair in patients who have had previous DTA repair.

In the most complex form of reoperation, namely repairing a failure of a previous open repair, a minimalist approach is taken in that only the problematic portion of the existing graft is repaired. For example, in cases of previous extent II TAAA repair in which a visceral patch aneurysm has formed, only the segment containing the visceral arteries typically is repaired. Although surgery for repair failure related to graft infection was uncommon in this series, infection necessitates the use of additional techniques, such as replacing the entire graft, extensive debridement, using pedicled omentum to cover the replacement graft, delivering antibiotics via irrigation catheters, or using a graft soaked in antibiotics (ie, prepared tableside by soaking a polyester graft in rifampin [600 mg/vial]). The use of a homograft, rather than a standard synthetic graft, in the distal aorta is not recommended. 


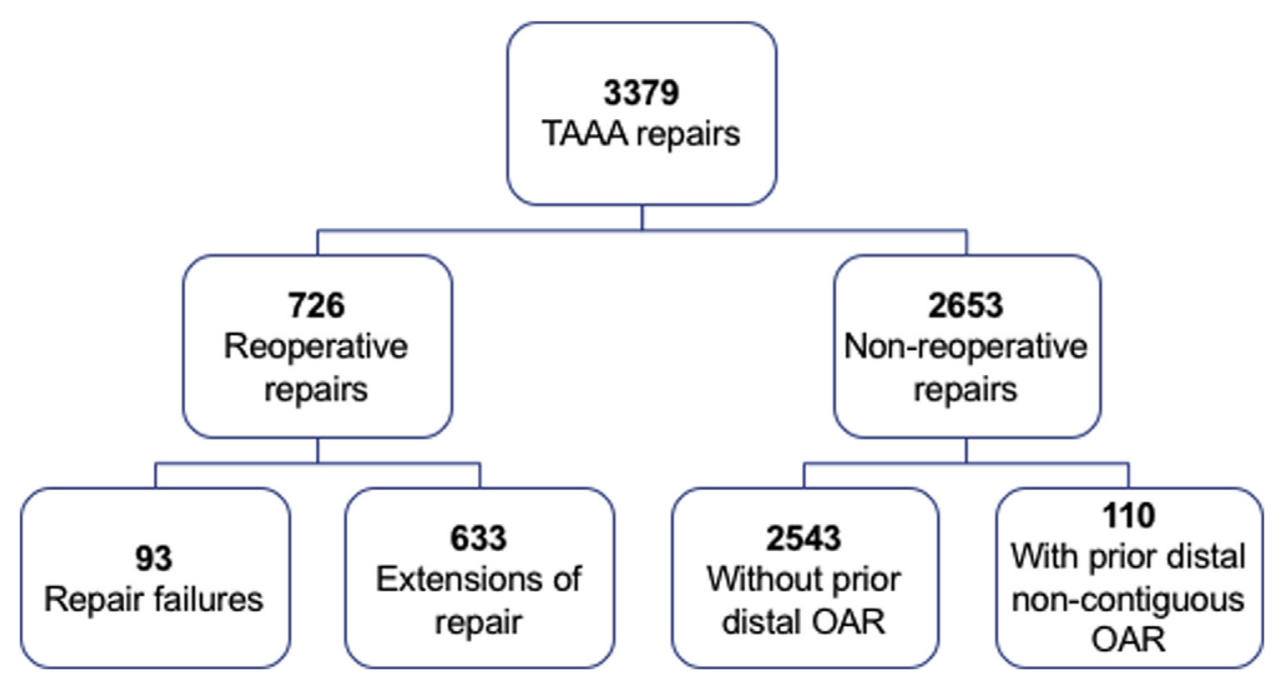

FIGURE 3. Schematic pathway shows the grouping of 3379 TAAA repairs by reoperative status and indication. Repair failure ( $\mathrm{n}=93)$ was defined as a complication of previous distal OAR; extension of repair $(n=633)$ was defined as a repair necessitated by the progression of disease into aortic segments that are adjacent to and contiguous with the previously repaired segment. The remaining 2653 repairs were defined as non-reoperative and included 110 extent I TAAA repairs that were noncontiguous with previous open infrarenal abdominal aortic repairs. TAAA, Thoracoabdominal aortic aneurysm; $O A R$, open aortic repair.

\section{Statistical Analysis and Data Presentation}

Statistical analyses were performed with SAS, version 9.4 (SAS Institute, Inc, Cary, NC), IBM SPSS Statistics 22 (IBM Corp, Armonk, NY), and R version 3.4.0 from The R Project for Statistical Computing (Vienna, Austria). Analyses were first performed to compare all reoperative patients to the non-reoperative patients. The reoperative patients also were analyzed separately according to their indication for repair (repair failure vs extension of repair). Continuous variables are presented as mean $\pm \mathrm{SD}$ or median (interquartile range), as appropriate. Categorical variables are presented as number and percentage. Bivariate comparisons were conducted with the Pearson $\chi^{2}$ test, Fisher exact test, or nonparametric Wilcoxon rank sum test, as appropriate.

To identify independent predictors of adverse event in the overall group of 3379 repairs, we built multivariable logistic regression models by entering clinically relevant preoperative and intraoperative factors that showed a univariate association with a $P$ value of $\leq .1$ into the model and then applied a backward selection method with a removal $P$ value of .05 ; reoperative status did not meet criteria for model entry and was forced into the model (these variables are listed in Table E1). We also performed an adjusted analysis of operative death and persistent stroke, renal failure

TABLE 1. Details regarding previous aortic repairs for patients stratified by reoperative status and indication

\begin{tabular}{|c|c|c|c|c|c|c|c|}
\hline \multirow[b]{2}{*}{ Variable } & \multirow[b]{2}{*}{$\begin{array}{c}\text { All } \\
(\mathrm{n}=\mathbf{3 3 7 9})\end{array}$} & \multicolumn{2}{|c|}{ Reoperative status } & \multirow[b]{2}{*}{$P$ value } & \multicolumn{2}{|c|}{ Reoperative indication } & \multirow[b]{2}{*}{$P$ value } \\
\hline & & $\begin{array}{l}\text { Reoperative } \\
(n=726)\end{array}$ & $\begin{array}{c}\text { Non-reoperative } \\
\quad(\mathbf{n}=\mathbf{2 6 5 3})^{*}\end{array}$ & & $\begin{array}{c}\text { Repair failure } \\
\quad(\mathbf{n}=93)\end{array}$ & $\begin{array}{l}\text { Extension of repair } \\
\quad(n=633)\end{array}$ & \\
\hline Previous aortic repair $\dagger$ & $1475(43.7)$ & $726(100)$ & 749 (28.2) & $<.001$ & $93(100)$ & $633(100)$ & - \\
\hline Proximal OAR & $772(22.8)$ & $146(20.1)$ & $626(23.6)$ & .05 & $34(36.6)$ & $112(17.7)$ & $<.001$ \\
\hline Distal OAR & $836(24.7)$ & $726(100)$ & $110(4.1)^{*}$ & $<.001$ & $93(100)$ & $633(100)$ & - \\
\hline DTA & $176(5.2)$ & $176(24.2)$ & 0 & - & $19(20.4)$ & $157(24.8)$ & .4 \\
\hline Extent I TAAA & 65 (1.9) & $65(9.0)$ & 0 & - & $16(17.2)$ & $49(7.7)$ & .006 \\
\hline Extent II TAAA & $35(1.1)$ & $35(4.8)$ & 0 & - & $35(37.6)$ & 0 & $<.001$ \\
\hline Extent III TAAA & $16(0.5)$ & $16(2.2)$ & 0 & - & $14(15.1)$ & $2(0.3)$ & $<.001$ \\
\hline Extent IV TAAA & $33(1.0)$ & $33(4.5)$ & 0 & - & $18(19.4)$ & $15(2.4)$ & $<.001$ \\
\hline Direct repair $\ddagger$ & $6(0.2)$ & $6(0.8)$ & 0 & - & 0 & $6(0.9)$ & 1 \\
\hline AAA & $579(17.1)$ & 469 (64.6) & $110(4.1)^{*}$ & - & $20(21.5)$ & 449 (70.9) & $<.001$ \\
\hline Infrarenal AAA & $563(16.7)$ & $453(62.4)$ & $110(4.1)^{*}$ & - & $17(18.3)$ & $436(68.9)$ & $<.001$ \\
\hline$\geq 2$ distal OAR & $75(2.2)$ & $75(10.3)$ & 0 & $<.001$ & $24(25.8)$ & $51(8.1)$ & $<.001$ \\
\hline Distal EAR & $75(2.2)$ & $13(1.8)$ & $62(2.3)$ & .4 & $3(3.2)$ & $10(1.6)$ & .3 \\
\hline
\end{tabular}

Data are presented as no. (\%). OAR, Open aortic repair; DTA, descending thoracic aorta; TAAA, thoracoabdominal aortic aneurysm; $A A A$, abdominal aortic aneurysm; $E A R$, endovascular aortic repair. *This group included 110 patients who underwent extent I TAAA repair after noncontiguous previous open infrarenal abdominal aortic repair and who did not meet criteria for reoperative repair. †Previous open or endovascular aortic repair of any section of the aorta. ‡Direct repair involves no graft replacement, is a patch repair, or is a TAAA repair of unknown extent. 
TABLE 2. Preoperative characteristics of patients stratified by reoperative status and indication

\begin{tabular}{|c|c|c|c|c|c|c|c|}
\hline \multirow[b]{2}{*}{ Variable } & \multirow[b]{2}{*}{$\begin{array}{c}\text { All } \\
(\mathbf{n}=\mathbf{3 3 7 9})\end{array}$} & \multicolumn{2}{|c|}{ Reoperative status } & \multirow[b]{2}{*}{$\begin{array}{c}P \\
\text { value }\end{array}$} & \multicolumn{2}{|c|}{ Reoperative indication } & \multirow[b]{2}{*}{$\begin{array}{c}P \\
\text { value }\end{array}$} \\
\hline & & $\begin{array}{c}\text { Reoperative } \\
(n=726)\end{array}$ & $\begin{array}{l}\text { Non-reoperative } \\
\quad(\mathbf{n}=\mathbf{2 6 5 3})^{*}\end{array}$ & & $\begin{array}{l}\text { Repair failure } \\
\quad(\mathbf{n}=93)\end{array}$ & $\begin{array}{l}\text { Extension of repair } \\
\qquad(\mathrm{n}=633)\end{array}$ & \\
\hline Age, y & $64[54-71]$ & $69[61-74]$ & $67[59-73]$ & .002 & $65[53-73]$ & $69[62-74]$ & .8 \\
\hline Age $\leq 50$ y & $451(13.3)$ & $88(12.1)$ & $363(13.7)$ & .3 & $19(20.4)$ & $69(10.9)$ & .009 \\
\hline Male sex & $2092(61.9)$ & $538(74.1)$ & $1554(58.6)$ & $<.001$ & $57(61.3)$ & $481(76.0)$ & .003 \\
\hline Connective tissue disorder & $326(9.6)$ & $86(11.8)$ & $240(9.0)$ & .02 & $24(25.8)$ & $62(9.8)$ & $<.001$ \\
\hline Marfan syndrome & $296(8.8)$ & $81(11.2)$ & $215(8.1)$ & .01 & $21(22.6)$ & $60(9.5)$ & $<.001$ \\
\hline Aortic aneurysm without dissection & $2157(63.8)$ & $496(68.3)$ & $1661(62.6)$ & .005 & $51(54.8)$ & $445(70.3)$ & .003 \\
\hline \multicolumn{8}{|l|}{ Aortic dissection } \\
\hline Acute or subacute aortic dissection & $170(5.0)$ & $20(2.8)$ & $150(5.7)$ & .002 & $4(4.3)$ & $16(2.5)$ & .3 \\
\hline Chronic aortic dissection & $1052(31.1)$ & $210(28.9)$ & $842(31.7)$ & .1 & $38(40.9)$ & $172(27.2)$ & .007 \\
\hline DeBakey type I aortic dissection & $443(13.1)$ & $69(9.5)$ & $374(14.1)$ & .001 & $15(16.1)$ & $54(8.5)$ & .02 \\
\hline DeBakey type III aortic dissection & $743(22.0)$ & $154(21.2)$ & $589(22.2)$ & .6 & $25(26.9)$ & $129(20.4)$ & .2 \\
\hline Maximum distal aortic diameter, $\mathrm{cm}$ & $\begin{array}{r}6.1[5.5-7.0] \\
(\mathrm{n}=2990)\end{array}$ & $\begin{array}{r}6.3[5.7-7.2] \\
(\mathrm{n}=665)\end{array}$ & $\begin{array}{l}6.2[5.5-7.2] \\
\quad(n=2325)\end{array}$ & .1 & $\begin{array}{c}7.0[6.0-8.0] \\
(\mathrm{n}=84)\end{array}$ & $\begin{array}{r}6.3[5.6-7.2] \\
\quad(n=581)\end{array}$ & .95 \\
\hline Diameter $>7.5 \mathrm{~cm}$ & $578(17.1)$ & $130(17.9)$ & $448(16.9)$ & .5 & $28(30.1)$ & $102(16.1)$ & .001 \\
\hline Hypertension & $2874(85.1)$ & $635(87.5)$ & $2239(84.4)$ & .04 & $81(87.1)$ & $554(87.5)$ & .9 \\
\hline Hyperlipidemia & $1009(29.9)$ & $239(32.9)$ & $770(29.0)$ & .04 & $30(32.3)$ & $209(33.0)$ & .9 \\
\hline Diabetes & $276(8.2)$ & $57(7.9)$ & $219(8.3)$ & .7 & $7(7.5)$ & $50(7.9)$ & .9 \\
\hline Coronary artery disease & $1134(33.6)$ & $311(42.8)$ & $823(31.0)$ & $<.001$ & $37(39.8)$ & $274(43.3)$ & .5 \\
\hline Previous CABG & $508(15.0)$ & $153(21.1)$ & $355(13.4)$ & $<.001$ & $11(11.8)$ & $142(22.4)$ & .02 \\
\hline Previous MI & $662(19.6)$ & $185(25.5)$ & $477(18.0)$ & $<.001$ & $25(26.9)$ & $160(25.3)$ & .7 \\
\hline Cerebrovascular disease & $589(17.4)$ & $176(24.2)$ & $413(15.6)$ & $<.001$ & $15(16.1)$ & $161(25.4)$ & .05 \\
\hline History of stroke & $346(10.2)$ & $105(14.5)$ & $241(9.1)$ & $<.001$ & $8(8.6)$ & $97(15.3)$ & .09 \\
\hline Peripheral vascular disease & $862(25.5)$ & $200(27.5)$ & $662(25.0)$ & .2 & $19(20.4)$ & $181(28.6)$ & .1 \\
\hline Chronic renal insufficiency $\dagger$ & $102(3.0)$ & $25(3.4)$ & $77(2.9)$ & .5 & $4(4.3)$ & $21(3.3)$ & .6 \\
\hline History of renal failure or dialysis & $65(1.9)$ & $19(2.6)$ & $46(1.7)$ & .1 & $3(3.2)$ & $16(2.5)$ & .7 \\
\hline eGFR & $\begin{array}{l}68[52-87] \\
(\mathrm{n}=3082)\end{array}$ & $\begin{array}{l}61[45-79] \\
(n=671)\end{array}$ & $\begin{array}{l}65[49-83] \\
(\mathrm{n}=2411)\end{array}$ & .008 & $\begin{array}{l}67[47-89] \\
(\mathrm{n}=86)\end{array}$ & $\begin{array}{l}60[44-78] \\
(n=585)\end{array}$ & .3 \\
\hline eGFR quartiles & & & & $<.001$ & & & .01 \\
\hline eGFR Q1 [4-40] & $506(15.0)$ & $143(19.7)$ & $363(13.7)$ & - & $16(17.2)$ & $127(20.1)$ & - \\
\hline eGFR Q2 [41-76] & $1606(47.5)$ & $342(47.1)$ & $1264(47.6)$ & - & 39 (41.9) & $303(47.9)$ & - \\
\hline eGFR Q3 [77-112] & $879(26.0)$ & $167(23.0)$ & $712(26.8)$ & - & $24(25.8)$ & 143 (22.6) & - \\
\hline eGFR Q4 [113-148] & $91(2.7)$ & $19(2.6)$ & $72(2.7)$ & - & $7(7.5)$ & $12(1.9)$ & - \\
\hline $\begin{array}{l}\text { History of COPD, emphysema, } \\
\text { or bronchitis }\end{array}$ & $1229(36.4)$ & $310(42.7)$ & 919 (34.6) & $<.001$ & $31(33.3)$ & $279(44.1)$ & .05 \\
\hline Past or current tobacco use & $2667(78.9)$ & $611(84.2)$ & $2056(77.5)$ & $<.001$ & $70(75.3)$ & $541(85.5)$ & .01 \\
\hline Asymptomatic & $1210(35.8)$ & $268(36.9)$ & $942(35.5)$ & .5 & $30(32.3)$ & $238(37.6)$ & .3 \\
\hline Symptomatic & $2169(64.2)$ & $458(63.1)$ & $1711(64.5)$ & .5 & $63(67.7)$ & $395(62.4)$ & .3 \\
\hline Acute symptoms & $562(16.6)$ & $113(15.6)$ & 449 (16.9) & .4 & $22(23.7)$ & $91(14.4)$ & .02 \\
\hline Chronic symptoms & $1705(50.5)$ & $357(49.2)$ & $1348(50.8)$ & .4 & $42(45.2)$ & $315(49.8)$ & .4 \\
\hline Rupture & $173(5.1)$ & $33(4.5)$ & $140(5.3)$ & .4 & $10(10.8)$ & $23(3.6)$ & .002 \\
\hline
\end{tabular}

Data are presented as no. (\%) or median [IQR]. $C A B G$, Coronary artery bypass grafting; $M I$, myocardial infarction; $e G F R$, estimated glomerular filtration rate; $Q$, quartile; $C O P D$, chronic obstructive pulmonary disease. *This group included 110 patients who underwent extent I TAAA repair after noncontiguous previous open infrarenal abdominal aortic repair and who did not meet criteria for reoperative repair. $\nmid$ Serum creatinine level $\geq 3.0 \mathrm{mg} / \mathrm{dL}$ or dialysis. 
TABLE 3. Operative details of repair stratified by reoperative status and indication

\begin{tabular}{|c|c|c|c|c|c|c|c|}
\hline \multirow[b]{2}{*}{ Variable } & \multirow[b]{2}{*}{$\begin{array}{c}\text { All } \\
(\mathbf{n}=\mathbf{3 3 7 9})\end{array}$} & \multicolumn{2}{|c|}{ Reoperative status } & \multirow[b]{2}{*}{$\begin{array}{c}P \\
\text { value }\end{array}$} & \multicolumn{2}{|c|}{ Reoperative indication } & \multirow[b]{2}{*}{$\begin{array}{c}P \\
\text { value }\end{array}$} \\
\hline & & $\begin{array}{c}\text { Reoperative } \\
(\mathbf{n}=\mathbf{7 2 6})\end{array}$ & $\begin{array}{c}\text { Non-reoperative } \\
\quad(\mathbf{n}=\mathbf{2 6 5 3})^{*}\end{array}$ & & $\begin{array}{c}\text { Repair failure } \\
\quad(\mathbf{n}=93)\end{array}$ & $\begin{array}{l}\text { Extension of repair } \\
\quad(n=633)\end{array}$ & \\
\hline Era of repair & & & & .07 & & & .06 \\
\hline Era 1 (1986-1998) & $1158(34.3)$ & $244(33.6)$ & $914(34.5)$ & - & $29(31.2)$ & $215(34.0)$ & - \\
\hline Era 2 (1999-2004) & $1059(31.3)$ & $252(34.7)$ & $807(30.4)$ & - & $42(45.2)$ & $210(33.2)$ & - \\
\hline Era 3 (2005-2016) & $1162(34.4)$ & $230(31.7)$ & $932(35.1)$ & - & $22(23.7)$ & $208(32.9)$ & - \\
\hline Urgency of operation & & & & .2 & & & .005 \\
\hline Elective & $2632(77.9)$ & $579(79.8)$ & $2053(77.4)$ & - & $64(68.8)$ & $515(81.4)$ & - \\
\hline Urgent or emergent & $747(22.1)$ & $147(20.2)$ & $600(22.6)$ & - & $29(31.2)$ & $118(18.6)$ & - \\
\hline Extent I & $933(27.6)$ & $27(3.7)$ & $906(34.2)$ & $<.001$ & $1(1.1)$ & $26(4.1)$ & .1 \\
\hline Extent II & $1089(32.2)$ & $191(26.3)$ & $898(33.8)$ & $<.001$ & $10(10.8)$ & $181(28.6)$ & $<.001$ \\
\hline Extent III & $678(20.1)$ & $277(38.2)$ & $401(15.1)$ & $<.001$ & $40(43.0)$ & $237(37.4)$ & .3 \\
\hline Extent IV & $679(20.1)$ & $231(31.8)$ & $448(16.9)$ & $<.001$ & $42(45.2)$ & $189(29.9)$ & .003 \\
\hline Reverse elephant trunk & $83(2.5)$ & $5(0.7)$ & $78(2.9)$ & .001 & $0(0)$ & $5(0.8)$ & .4 \\
\hline Elephant trunk completion repair & $143(4.2)$ & $3(0.4)$ & $140(5.3)$ & $<.001$ & $0(0)$ & $3(0.5)$ & .5 \\
\hline Extraction of endograft & $57(1.7)$ & $8(1.1)$ & $49(1.8)$ & .2 & $3(3.2)$ & $5(0.8)$ & .03 \\
\hline Clamping proximal to left subclavian artery & $536(15.9)$ & $37(5.1)$ & $499(18.8)$ & $<.001$ & $1(1.1)$ & $36(5.7)$ & .06 \\
\hline Intercostal/lumbar artery reattachment & $1624(48.1)$ & $271(37.3)$ & $1353(51.0)$ & $<.001$ & $20(21.5)$ & $251(39.7)$ & .001 \\
\hline Splenectomy & $405(12.0)$ & $151(20.8)$ & $254(9.6)$ & $<.001$ & $47(50.5)$ & $104(16.4)$ & $<.001$ \\
\hline Aortic clamp time, min & $50[38-66]$ & 50 [39-67] & 50 [37-66] & .6 & $50[39-70]$ & $50[39-66]$ & .8 \\
\hline \multicolumn{8}{|l|}{ Management of visceral/renal arteries } \\
\hline Bypass graft & $859(25.4)$ & $308(42.4)$ & $551(20.8)$ & $<.001$ & $48(51.6)$ & $260(41.1)$ & .06 \\
\hline Endarterectomy & $877(26.0)$ & $247(34.0)$ & $630(23.7)$ & $<.001$ & $21(22.6)$ & $226(35.7)$ & .01 \\
\hline Stenting & $272(8.0)$ & $84(11.6)$ & $188(7.1)$ & $<.001$ & $9(9.7)$ & $75(11.8)$ & .5 \\
\hline Endarterectomy, stenting, or bypass & $1400(41.4)$ & $429(59.1)$ & $971(36.6)$ & $<.001$ & $56(60.2)$ & $373(58.9)$ & .8 \\
\hline \multicolumn{8}{|l|}{ Adjuncts } \\
\hline Cerebrospinal fluid drainage & 1574 (46.6) & $314(43.3)$ & $1260(47.5)$ & .04 & $40(43.0)$ & $274(43.3)$ & .96 \\
\hline Left heart bypass & $1521(45.0)$ & $163(22.5)$ & $1358(51.2)$ & $<.001$ & $2(2.2)$ & $161(25.4)$ & $<.001$ \\
\hline Left heart bypass time, min & $\begin{array}{c}24[19-29] \\
(\mathrm{n}=1503)\end{array}$ & $\begin{array}{c}21[17-27] \\
\quad(\mathrm{n}=159)\end{array}$ & $\begin{array}{c}24[19-30] \\
(\mathrm{n}=1344)\end{array}$ & $<.001$ & $\begin{array}{r}20[16-24] \\
\quad(\mathrm{n}=2)\end{array}$ & $\begin{array}{l}21[17-27] \\
\quad(\mathrm{n}=157)\end{array}$ & .5 \\
\hline Hypothermic circulatory arrest & $52(1.5)$ & $5(0.7)$ & $47(1.8)$ & .03 & $3(3.2)$ & $2(0.3)$ & .002 \\
\hline Cold renal perfusion & $1988(58.8)$ & $540(74.3)$ & $1448(54.6)$ & $<.001$ & $67(72.0)$ & $473(74.7)$ & .6 \\
\hline Selective perfusion of visceral arteries & $802(23.7)$ & $108(14.9)$ & $694(26.2)$ & $<.001$ & $1(1.1)$ & $107(16.9)$ & $<.001$ \\
\hline
\end{tabular}

Data are presented as $\mathrm{n}(\%)$ or median [IQR]. *This group included 110 patients who underwent extent I TAAA repair after non-contiguous previous open infrarenal abdominal aortic repair and who did not meet criteria for reoperative repair.

necessitating dialysis, and paraparesis or paraplegia among the extent II repairs to compare relative risk by reoperative status. Postoperative survival was first estimated by the Kaplan-Meier method; the log-rank test was used to differentiate between groups. We then built a Cox proportional hazards model to adjust survival for clinically relevant covariates: patient age at repair, coronary artery disease, cerebrovascular disease, and past or current tobacco use. For this model, we used a backward selection method and a removal $P$ value of .05. The assumption of proportional hazards was examined based on weighted Schoenfeld residuals.

\section{RESULTS}

\section{Preoperative Characteristics}

Preoperative characteristics are presented in Table 2. There were several notable differences between reoperative and non-reoperative repair patients. There were also substantial differences between patients who underwent surgery for repair failure versus progressively worsening aortic disease. The extension-of-repair cohort was older than both the repair-failure group and the non-reoperative group. This cohort also had greater preoperative prevalence of several comorbidities, including a history of coronary artery disease, cerebrovascular disease, peripheral vascular disease, and chronic obstructive pulmonary disease or bronchitis; moreover, these patients were more likely to be male, to have an aneurysm unrelated to dissection, and to be current or former smokers.

The repair-failure group was younger than both the extension-of-repair and the non-reoperative groups, and it 
TABLE 4. Early outcomes for patients stratified by reoperative status and indication

\begin{tabular}{|c|c|c|c|c|c|c|c|}
\hline \multirow[b]{2}{*}{ Variable } & \multirow[b]{2}{*}{$\begin{array}{c}\text { All } \\
(\mathrm{n}=\mathbf{3 3 7 9})\end{array}$} & \multicolumn{2}{|c|}{ Reoperative status } & \multirow[b]{2}{*}{$\begin{array}{c}P \\
\text { value }\end{array}$} & \multicolumn{2}{|c|}{ Reoperative indication } & \multirow[b]{2}{*}{$\begin{array}{c}P \\
\text { value }\end{array}$} \\
\hline & & $\begin{array}{l}\text { Reoperative } \\
(n=726)\end{array}$ & $\begin{array}{l}\text { Non-reoperative } \\
\quad(\mathbf{n}=\mathbf{2 6 5 3})^{*}\end{array}$ & & $\begin{array}{l}\text { Repair failure } \\
\quad(\mathbf{n}=93)\end{array}$ & $\begin{array}{c}\text { Extension of repair } \\
\quad(n=633)\end{array}$ & \\
\hline Adverse event $\dagger$ & 487 (14.4) & $96(13.2)$ & $391(14.7)$ & .3 & $17(18.3)$ & 79 (12.5) & .1 \\
\hline Operative death & $253(7.5)$ & $59(8.1)$ & $194(7.3)$ & .5 & $10(10.8)$ & $49(7.7)$ & .3 \\
\hline In-hospital & $240(7.1)$ & $56(7.7)$ & $184(6.9)$ & .5 & $9(9.7)$ & $47(7.4)$ & .4 \\
\hline $30-d$ & $163(4.8)$ & $34(4.7)$ & $129(4.9)$ & .8 & $6(6.5)$ & $28(4.4)$ & .4 \\
\hline Stroke & $106(3.1)$ & $21(2.9)$ & $85(3.2)$ & .7 & $4(4.3)$ & $17(2.7)$ & .4 \\
\hline Persistent stroke $\ddagger$ & $79(2.3)$ & $15(2.1)$ & $64(2.4)$ & .6 & $3(3.2)$ & $12(1.9)$ & .4 \\
\hline Spinal cord deficit & $325(9.6)$ & $58(8.0)$ & $267(10.1)$ & .09 & $5(5.4)$ & $53(8.4)$ & .3 \\
\hline Persistent paraplegia & $101(3.0)$ & $23(3.2)$ & $78(2.9)$ & .7 & $2(2.2)$ & $21(3.3)$ & .5 \\
\hline Persistent paraparesis $\Varangle$ & $82(2.4)$ & $12(1.7)$ & $70(2.6)$ & .1 & $1(1.1)$ & $11(1.7)$ & .6 \\
\hline Acute renal dysfunction & $412(12.2)$ & $87(12.0)$ & $325(12.3)$ & .8 & $17(18.3)$ & $70(11.1)$ & .045 \\
\hline Renal failure necessitating dialysis & $254(7.5)$ & $53(7.3)$ & $201(7.6)$ & .8 & $12(12.9)$ & $41(6.5)$ & .03 \\
\hline Persistent renal failure & $192(5.7)$ & $41(5.6)$ & $151(5.7)$ & .96 & $9(9.7)$ & $32(5.1)$ & .07 \\
\hline Cardiac complication & $879(26.0)$ & $178(24.5)$ & $701(26.4)$ & .3 & $21(22.6)$ & $157(24.8)$ & .6 \\
\hline Atrial arrhythmia & $553(16.4)$ & $105(14.5)$ & $448(16.9)$ & .1 & $8(8.6)$ & $97(15.3)$ & .09 \\
\hline Pulmonary complication & $1208(35.8)$ & $248(34.2)$ & $960(36.2)$ & .3 & $27(29.0)$ & $221(34.9)$ & .3 \\
\hline Respiratory failure & $840(24.9)$ & $180(24.8)$ & $660(24.9)$ & .98 & $23(24.7)$ & $158(25.0)$ & .96 \\
\hline Tracheostomy & $288(8.5)$ & $58(8.0)$ & $230(8.7)$ & 6 & $9(9.7)$ & 49 (7.7) & .5 \\
\hline Left vocal cord paralysis & $520(15.4)$ & $56(7.7)$ & $464(17.5)$ & $<.001$ & $4(4.3)$ & $52(8.2)$ & .2 \\
\hline Bleeding requiring reoperation & $112(3.3)$ & $25(3.4)$ & $87(3.3)$ & .8 & $1(1.1)$ & $24(3.8)$ & .2 \\
\hline Early survivors & $(\mathrm{n}=3126)$ & $(\mathrm{n}=667)$ & $(\mathrm{n}=2459)$ & & $(\mathrm{n}=83)$ & $(\mathrm{n}=584)$ & \\
\hline $\begin{array}{l}\text { Discharge with life-altering } \\
\text { complication } \S\end{array}$ & $234(7.5)$ & $37(5.5)$ & $197(8.0)$ & .03 & $7(8.4)$ & $30(5.1)$ & .2 \\
\hline Length of ICU stay, d & $\begin{array}{c}4[3-8] \\
(\mathrm{n}=3029)\end{array}$ & $4[2.25-6]$ & $\begin{array}{c}4[3-7] \\
(\mathrm{n}=2381)\end{array}$ & .07 & $\begin{array}{l}4[3-6] \\
(n=82)\end{array}$ & $\begin{array}{c}4[2-6] \\
(n=566)\end{array}$ & .3 \\
\hline Length of hospital stay, $d$ & $\begin{array}{c}12[9-17] \\
(\mathrm{n}=3118)\end{array}$ & $11[9-15]$ & $\begin{array}{c}12[9-17] \\
(\mathrm{n}=2453)\end{array}$ & .001 & $\begin{array}{l}10[9-14] \\
(\mathrm{n}=82)\end{array}$ & $\begin{array}{l}11[9-15] \\
(\mathrm{n}=583)\end{array}$ & .8 \\
\hline
\end{tabular}

Data are presented as $\mathrm{n}(\%)$ or median [IQR]. ICU, Intensive care unit. *This group included 110 patients who underwent extent I TAAA repair after noncontiguous previous open infrarenal abdominal aortic repair and who did not meet criteria for reoperative repair. $\dagger$ Adverse event is defined as operative death or discharge with renal failure necessitating dialysis, paraplegia, paraparesis, or stroke. †Persistent is defined as present at the time of operative death or discharge. §Discharged with renal failure necessitating dialysis, paraplegia, paraparesis, or stroke.

had the greatest rates of connective tissue disorders and chronic aortic dissection; additionally, more patients in this group had large aneurysms. Although there was little difference among the groups regarding the presence of overall aortic symptoms, patients with repair failure had a high rate of aortic rupture-nearly 3 times greater than in the extension-of-repair group and more than twice as great as in the non-reoperative group.

In our overall group of 3379 repairs, previous repair of any section of the aorta was fairly common (43.6\%). Regarding previous distal open aortic repair, procedures in the repair-failure group were fairly well distributed across the distal aorta, extent II repair being the most common previous procedure. In contrast, in the extension-of-repair group, $68.9 \%$ of the previous repairs were for infrarenal AAA; as expected, there were no previous extent II repairs.

\section{Operative Details}

Operative details are presented in Table 3. Unsurprisingly, reoperative patients with repair failure more frequently underwent urgent or emergent repair than the extension-of-repair and non-reoperative patients. In addition, those with repair failure typically underwent less extensive (ie, extent III or IV) TAAA repair. Of note, reoperative patients infrequently underwent extent I repair. The most common reoperative procedure was extent III repair $(277 / 726 ; 38.1 \%)$, which was less than one half as frequent in the non-reoperative group $(401 / 2653 ; 15.1 \%)$. Regarding the management of visceral and renal arteries, more than one half of reoperative repairs involved endarterectomy, stenting, or bypass procedures, as compared with roughly one third of non-reoperative repairs. The rate of visceral or renal artery endarterectomy was greatest in the extension-of-repair cohort. CSF drainage 
TABLE 5. Multivariable analysis of predictors of adverse events after 3379 TAAA repairs*

\begin{tabular}{|c|c|c|}
\hline Variables & Odds ratio $(\mathrm{CI})$ & $P$ value \\
\hline Reoperation & & $.03 \dagger$ \\
\hline $\begin{array}{l}\text { Extension of repair vs } \\
\text { non-reoperative repair }\end{array}$ & $0.70(0.53-0.92)$ & - \\
\hline $\begin{array}{l}\text { Repair failure vs } \\
\text { non-reoperative repair }\end{array}$ & $1.14(0.64-2.04)$ & - \\
\hline Extension of repair vs repair failure & $0.62(0.33-1.14)$ & - \\
\hline Rupture & $2.16(1.46-3.20)$ & $<.001$ \\
\hline Extent II TAAA repair & $2.01(1.63-2.48)$ & $<.001$ \\
\hline Chronic renal insufficiency & $2.00(1.25-3.22)$ & .004 \\
\hline Splenectomy & $1.52(1.14-2.02)$ & .004 \\
\hline Endarterectomy, stenting, or bypass & $1.47(1.20-1.81)$ & $<.001$ \\
\hline Urgent or emergent repair & $1.34(1.03-1.73)$ & .03 \\
\hline Previous myocardial infarction & $1.29(1.01-1.63)$ & .04 \\
\hline Symptomatic & $1.27(1.01-1.60)$ & .04 \\
\hline Increasing patient age at repair, per year & $1.04(1.03-1.05)$ & $<.001$ \\
\hline
\end{tabular}

and cold renal perfusion were used liberally in reoperative repairs, but left heart bypass was used infrequently in the reoperative patients-probably because they had less extensive repairs. Notably, in the repair-failure cohort, more than half of the reoperations necessitated a splenectomy; this was probably related to dense adhesions contributing to a splenic tear that was too large to be repaired.

\section{Early Outcomes}

Interestingly, compared with the non-reoperative group, neither the reoperative group as a whole nor the repair-failure or extension-of-repair subgroup had a significantly higher rate of operative death. In addition, there were no substantial intergroup differences in the rates of persistent (ie, present at discharge) renal failure, paraplegia, paraparesis, or stroke, or the composite endpoint-adverse event (Table 4). Of the postoperative outcome variables examined, there were few substantial differences among the groups. In addition, multivariable analysis of adverse event rates (Table 5) for the full cohort did not associate reoperative repair with greater risk than primary repair; in fact, patients in the extension-of-repair group were less likely to have an adverse event than patients in the nonreoperative group. The strongest predictors (odds ratio $\geq 2$ ) of adverse event were rupture, extent II TAAA repair, and chronic renal insufficiency.
Regarding extent II repair, we compared 181 such procedures in the extension-of-repair group to 898 in the non-reoperative group (Table 6). Although there were differences in several preoperative and operative variables, there were no important differences between rates of key outcomes. Likewise, multivariable analysis of 1089 extent II repairs did not identify reoperation as predictive of key postoperative complications (Table 7).

\section{Midterm Outcomes}

For the 3126 patients who survived to hospital discharge, the median clinical follow-up time was 5.6 years [interquartile range: 2.5-9.7 years]. Although we identified few differences between the reoperative and non-reoperative groups regarding early outcomes, Kaplan-Meier survival analysis showed that, over time, the reoperative groups fared significantly worse than the non-reoperative group $(P<.001, \log$-rank test; Figure 4, $A)$; 10 -year survival was estimated to be $23.9 \% \pm 4.9 \%$ for patients with repair failure and $28.4 \% \pm 2.0 \%$ for those with extension of repair, whereas it was $40.1 \% \pm 1.1 \%$ for those with non-reoperative repair. In addition, after adjustment were made for age, coronary artery disease, cerebrovascular disease, and past or current tobacco use in a Cox regression survival analysis, the patients with non-reoperative repairs continued to fare better over time $(P<.001$; Figure 4, $B$; Table 8$)$.

\section{DISCUSSION}

Despite the added complexity of reoperative TAAA surgery, our experience suggests that it is not associated with a greater risk of paraplegia, operative death, or other complications (Table 5); this apparently holds true even for the most extensive reoperative TAAA repairs, extent II repairs (Table 6). The reason could be related to the use of a more cautious surgical approach during reoperations so as to minimize further operative trauma. Like us, Kawaharada and colleagues ${ }^{13}$ reported no differences in the rates of early mortality, paraplegia, paraparesis, or renal failure in patients with TAAA who had undergone previous DTA repair and those who had not undergone previous repair. Our earlier report ${ }^{20}$ concerning TAAA repair after a broader category of reoperations generally supports our current results; there, we speculated that the factors that enabled patients to withstand the stress of the initial aortic repair also conferred a late benefit on survivors who needed additional repair.

In contrast, Lombardi and colleagues ${ }^{4}$ found that although previous AAA or TAAA repair was not associated with greater mortality in TAAA repair patients, previous AAA repair was associated with greater morbidity (arrhythmia, renal failure, pulmonary complications, and paraplegia). Likewise, Schlosser and colleagues ${ }^{5}$ found 
TABLE 6. Key preoperative characteristics, operative details, and outcomes after 1089 extent II TAAA repairs

\begin{tabular}{|c|c|c|c|c|c|}
\hline Variables & $\begin{array}{c}\text { All } \\
(\mathbf{n}=\mathbf{1 0 8 9})\end{array}$ & $\begin{array}{l}\text { Non-reoperative } \\
\quad(\mathbf{n}=\mathbf{8 9 8})^{*}\end{array}$ & $\begin{array}{l}\text { Extension of repair } \\
\quad(n=181)\end{array}$ & $\boldsymbol{P}$ value $\dagger$ & $\begin{array}{c}\text { Repair failure } \\
\quad(\mathbf{n}=\mathbf{1 0})\end{array}$ \\
\hline \multicolumn{6}{|l|}{ Preoperative characteristics } \\
\hline Age, y & 66 [56-72] & 65 [54-71] & 68 [61-74] & .009 & 70 [66-73] \\
\hline Connective tissue disorder & $176(16.2)$ & $153(17.0)$ & $22(12.2)$ & .1 & $1(10)$ \\
\hline Acute or subacute dissection & $60(5.5)$ & $51(5.7)$ & $8(4.4)$ & .6 & $1(10)$ \\
\hline Chronic dissection & $484(44.4)$ & $421(46.9)$ & $60(33.1)$ & .001 & $3(30)$ \\
\hline DeBakey type I aortic dissection & $223(20.5)$ & $205(22.8)$ & $17(9.4)$ & $<.001$ & $1(10)$ \\
\hline DeBakey type III aortic dissection & $311(28.6)$ & $259(28.8)$ & $49(27.1)$ & .7 & $3(30)$ \\
\hline Chronic renal insufficiency & $28(2.6)$ & $23(2.6)$ & $5(2.8)$ & .8 & 0 \\
\hline Previous myocardial infarction & $183(16.8)$ & $134(14.9)$ & $45(24.9)$ & .002 & $4(40)$ \\
\hline Symptomatic & $681(62.5)$ & $562(62.6)$ & $110(60.8)$ & .7 & $9(90)$ \\
\hline Diameter $>7.5 \mathrm{~cm}$ & $173(15.9)$ & $134(18.3)$ & $34(18.8)$ & .9 & $5(50)$ \\
\hline Rupture & $32(2.9)$ & $30(3.3)$ & $2(1.1)$ & .1 & 0 \\
\hline \multicolumn{6}{|l|}{ Operative details } \\
\hline Urgent or emergent repair & 203 (18.6) & $167(18.6)$ & $30(16.6)$ & .6 & $6(60)$ \\
\hline Intercostal/lumbar artery reattachment & $940(86.3)$ & $777(86.5)$ & $156(86.2)$ & .9 & $7(70)$ \\
\hline Splenectomy & $115(10.6)$ & $84(9.4)$ & $28(15.5)$ & .02 & $3(30)$ \\
\hline \multicolumn{6}{|l|}{ Management of visceral/renal arteries } \\
\hline Bypass graft & $348(32.0)$ & $269(30.0)$ & $75(41.4)$ & .003 & $4(40)$ \\
\hline Endarterectomy & $280(25.7)$ & $219(24.4)$ & $60(33.1)$ & .02 & $1(10)$ \\
\hline Stenting & $90(8.3)$ & $67(7.5)$ & $23(12.7)$ & .03 & 0 \\
\hline Endarterectomy, stenting, or bypass & $524(48.1)$ & $410(45.7)$ & $110(60.8)$ & $<.001$ & $4(40)$ \\
\hline \multicolumn{6}{|l|}{ Adjuncts } \\
\hline Cerebrospinal fluid drainage & $678(62.3)$ & $568(63.3)$ & $107(59.1)$ & .3 & $3(30)$ \\
\hline Left heart bypass & $898(82.5)$ & $761(84.7)$ & $135(74.6)$ & .002 & $2(20)$ \\
\hline Hypothermic circulatory arrest & $18(1.7)$ & $15(1.7)$ & $2(1.1)$ & .8 & $1(10)$ \\
\hline Cold renal perfusion & $723(66.4)$ & $597(66.5)$ & $118(65.2)$ & .7 & $8(80)$ \\
\hline Selective perfusion of visceral arteries & $695(63.8)$ & $598(66.6)$ & $96(53.0)$ & .001 & $1(10)$ \\
\hline \multicolumn{6}{|l|}{ Outcomes } \\
\hline Adverse event $\ddagger$ & 204 (18.7) & $164(18.3)$ & $35(19.3)$ & .8 & $5(50)$ \\
\hline Operative death & $101(9.3)$ & $78(8.7)$ & $22(12.2)$ & .2 & $1(10)$ \\
\hline Persistent stroke $\S$ & $37(3.4)$ & $28(3.1)$ & $8(4.4)$ & .4 & $1(10)$ \\
\hline Persistent paraplegia $\S$ & $51(4.7)$ & $38(4.2)$ & $11(6.1)$ & .3 & $2(20)$ \\
\hline Persistent paraparesis $\S$ & $35(3.2)$ & $30(3.3)$ & $5(2.8)$ & .8 & 0 \\
\hline Persistent renal failure $\S$ & $79(7.3)$ & $66(7.3)$ & $12(6.6)$ & .9 & $1(10)$ \\
\hline Bleeding necessitating return to $\mathrm{OR}$ & $40(3.7)$ & $31(3.5)$ & $9(5.0)$ & .4 & 0 \\
\hline
\end{tabular}

Data are presented as no. $(\%)$ or median [IQR]. OR, Operating room. $*$ This group included 110 patients who underwent extent I TAAA repair after noncontiguous previous open infrarenal abdominal aortic repair and who did not meet criteria for reoperative repair. $\dagger P$ value for the comparative analysis between the non-reoperative and extension-of-repair groups; the repair-failure group was not included in this analysis because of the small size $(\mathrm{n}=10)$ of this group. $\ddagger$ Adverse event is defined as operative death or discharge with renal failure necessitating dialysis, paraplegia, paraparesis, or stroke. §Persistent is defined as present at the time of operative death or discharge.

that the risk of postoperative renal failure after TAAA repair was greater in patients with previous AAA repair than in patients without AAA repair, but there was no difference in early mortality.

Our mid-term survival results revealed definitively that all patients tend to fare poorly by the 10-year time point; this could be related to their relatively advanced age at repair, which is often in the mid-to-late 60s. Notably, the reoperative groups had significantly worse mid-term survival than the non-reoperative group. Thus, although we detected few significant differences in early outcomes between groups, the long-term implications of reoperative repair warrant further study. Regarding long-term survival, a Dutch series of 500 TAAA repairs revealed a 10-year survival rate of $34 \%$, which is comparable with our findings in the non-reoperative group; of interest, this survival rate was substantially worse than that of a Dutch population matched by calendar year, age, and sex $(85 \%){ }^{2}$

Notably, when we categorized reoperative repairs according to whether they were performed to treat failure of a previous open repair or progression of aortic disease into a contiguous section of the aorta, we found several 
TABLE 7. Multivariable analysis of early outcomes after extent II thoracoabdominal aortic aneurysm repair $(n=1089)$

\begin{tabular}{llc}
\hline \multicolumn{1}{c}{ Variables } & Odds ratio (CI) & $\boldsymbol{P}$ value* \\
\hline Operative death & & .7 \\
Extension of repair vs non-reoperative & $1.22(0.72-2.08)$ & - \\
Repair failure vs non-reoperative & $0.75(0.09-6.13)$ & - \\
Extension of repair vs repair failure & $1.63(0.18-13.99)$ & - \\
Persistent stroke $\dagger$ & & .6 \\
Extension of repair vs non-reoperative & $1.28(0.57-2.88)$ & - \\
Repair failure vs non-reoperative & $2.84(0.34-24.02)$ & - \\
Extension of repair vs repair failure & $0.45(0.05-4.13)$ & - \\
Persistent renal failure requiring dialysis $\dagger$ & & .6 \\
Extension of repair vs non-reoperative & $0.72(0.37-1.39)$ & - \\
Repair failure vs non-reoperative & $1.18(0.14-9.77)$ & - \\
Extension of repair vs repair failure & $0.61(0.07-5.34)$ & - \\
Persistent paraparesis or paraplegia $\dagger$ & & .7 \\
Extension of repair vs non-reoperative & $1.07(0.60-1.94)$ & - \\
Repair failure vs non-reoperative & $2.01(0.39-10.45)$ & - \\
Extension of repair vs repair failure & $0.54(0.10-2.95)$ & - \\
\hline$C I$, Confidence interval. *Reflects a 3-way comparison of extension of repair, repair \\
failure, and non-reoperative repair. $\dagger$ Persistent is defined as present at the time of \\
operative death or hospital discharge.
\end{tabular}

differences in preoperative characteristics. For example, in the repair-failure reoperative group (in which the failures comprised mostly pseudoaneurysms and patch aneurysms), we found younger patients, with greater rates of aortic dissection, connective tissue disorders, and rupture. In addition, nearly one third of patients with repair failure presented for reoperation with large aneurysms $(>7.5 \mathrm{~cm})$ that were well beyond the guideline-based threshold for repair. $^{22}$ This suggests that despite the well-established late risk of reoperation in young patients, especially those with connective tissue disorders such as Marfan syndrome, ${ }^{14}$ adherence to postoperative surveillance protocols must be improved.

In contrast, the preoperative characteristics of our extension-of-repair group were different than we originally envisioned. We surmised that this group might have high rates of chronic aortic dissection, but in fact it had a lower rate of such dissection than did either the failure group or the non-reoperative group. In addition, in the extensionof-repair group, we found greater rates of comorbidities commonly associated with the atherosclerotic process: older age, coronary artery disease, cerebrovascular disease, and peripheral vascular disease, as well as a history of smoking. Although in our previously published series ${ }^{20}$ we found reoperative patients to be older, with elevated rates of coronary artery, cerebrovascular, and other disease, more than $40 \%$ of those patients had chronic aortic dissection, whereas only $27 \%$ of the patients in our current study had chronic aortic dissection.

In addition, nearly $70 \%$ of the current series' extensionof-repair group had previous infrarenal AAA repair. This indicates that some persons treated for infrarenal abdominal aortic disease remain at risk for thoracic and thoracoabdominal aortic disease. ${ }^{15}$ Because the majority of these previous abdominal aortic repairs were done outside our facility, it is difficult to determine how many such patients go on to need TAAA repair. Also, we do not know how the shift in practice to endovascular AAA repair will affect the outcomes of reoperative TAAA repair. For extension-of-repair patients, in our experience, it appears that surveillance protocols might be effective: The rate of rupture was low, there were relatively few large aneurysms, and $38 \%$ of patients were asymptomatic.
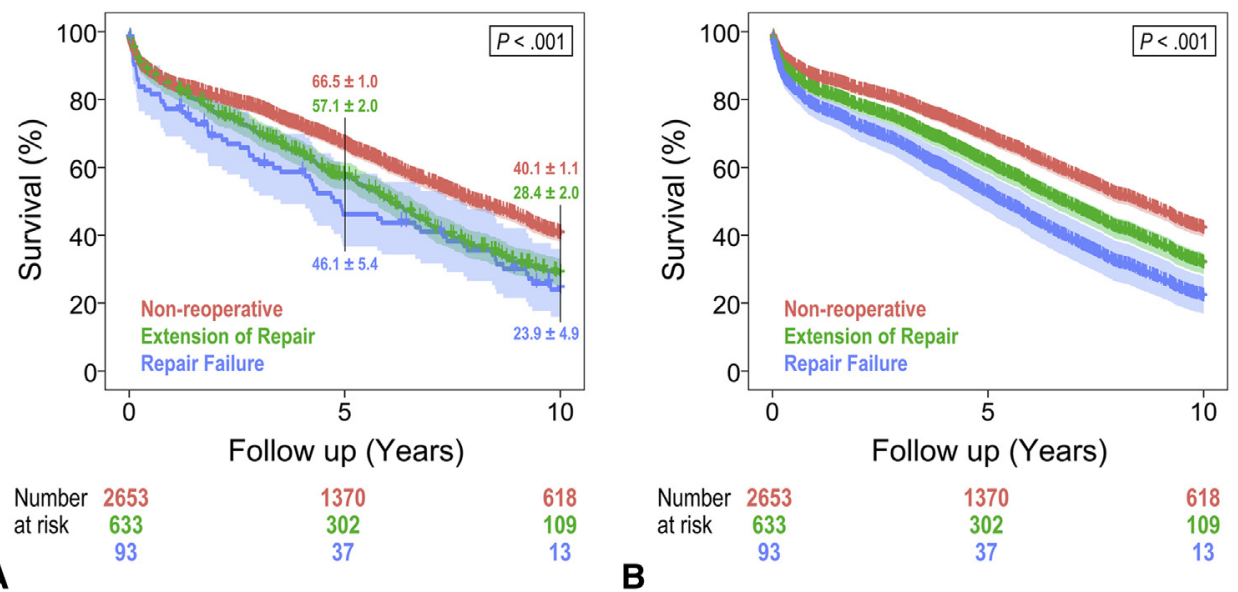

FIGURE 4. A, Kaplan-Meier curves of estimated survival after 3379 thoracoabdominal aortic aneurysm repairs stratified by reoperative status (non-reoperative repair, extension of repair, and repair failure), indicating a survival advantage for those patients who underwent non-reoperative repair $(P<.001)$. B, When we adjusted for age, coronary artery disease, cerebrovascular disease, and past or current tobacco use by using a Cox regression model to analyze survival after TAAA repair stratified by reoperative status, the non-reoperative repair patients' survival advantage remained $(P<.001)$. Survival curves were formulated from all patients' data and include 253 operative deaths. Confidence intervals are shown by the shaded bands. The corresponding hazard ratios, $95 \%$ confidence intervals, and $P$ values for panel B are shown in Table 8. 
TABLE 8. Cox regression analysis of death after 3379 TAAA repairs, adjusted for age (see Figure 4, B, for graphical interpretation)

\begin{tabular}{lccc}
\hline \multicolumn{1}{c}{ Variables } & Hazard ratio & $\mathbf{9 5 \%}$ CI & $\boldsymbol{P}$ value \\
\hline $\begin{array}{l}\text { Reoperation } \\
\text { Repair failure vs } \\
\quad \text { non-reoperative repair } \\
\begin{array}{l}\text { Extension of repair vs } \\
\quad \text { non-reoperative repair }\end{array}\end{array}$ & 1.75 & $(1.36-2.25)$ & - \\
$\begin{array}{l}\text { Increasing patient } \\
\text { age at repair, per year }\end{array}$ & 1.05 & $(1.001-1.37)$ & - \\
$\begin{array}{l}\text { Coronary artery disease } \\
\text { Cerebrovascular disease }\end{array}$ & 1.18 & $(1.07-1.30)$ & $<.001$ \\
\hline Past or current tobacco use & 1.36 & $(1.21-1.53)$ & $<.001$ \\
\hline
\end{tabular}

$C I$, Confidence interval.

Our findings represent the experience of a single practice led by the senior author (J.S.C.), which may differ substantially from other surgeons and centers. Limitations of our study include the fact that data were collected both retrospectively and prospectively from patients treated over a nearly 3-decade period, which we have previously speculated limits our ability to examine differences among surgical eras. ${ }^{1,18}$ Moreover, because our cohort was limited to patients who presented to our tertiary care center, the incidence of distal aortic open repair failure could not be determined definitively from our data. In addition, although we believe it is useful to categorize reoperative repair on the basis of repair failure or the type of previous repair, it is possible that the 110 non-reoperative procedures with previous open AAA repair (in which the previous AAA repair was not contiguous with the current TAAA repair and thus was categorized as non-reoperative repair) could in time cross over into the extension-of-repair cohort, which would alter the results presented here. Moreover, the small size of the repair-failure group $(n=93)$ limited the power of statistical analysis in comparing that group's outcomes to those of the other groups.

In conclusion, although reoperative TAAA surgery is more complicated than primary repair, the early postoperative results do not appear to be substantially different. However, multivariate analysis of early adverse event rates found less risk in the extension-of-repair group than in the non-reoperative repair group. Finally, midterm results indicate worse survival for patients who undergo reoperative surgery.

\section{Conflicts of Interest Statement}

Dr Coselli participates in clinical research trials conducted by Glaxo Smith Kline, Edwards Lifesciences, and Bolton Medical; consults for, receives royalties and a departmental educational grant from, and participates in clinical trials for Vascutek Terumo; and consults and participates in clinical trials for Medtronic, Inc, and WL Gore $\&$ Associates. Dr LeMaire participates in clinical trials for Medtronic, Inc, WL Gore \& Associates, and Vascutek Terumo, and consults for Vascutek Terumo. All other authors have nothing to disclose with regard to commercial support.

The authors thank several members of the Surgical Research Core of the Michael E. DeBakey Department of Surgery at Baylor College of Medicine, including Laurie Fondren for managing patient enrollment, and Scott A. Weldon, MA, CMI, for creating illustrations and figures. We also thank the Section of Scientific Publications at the Texas Heart Institute for providing editorial support.

\section{References}

1. Coselli JS, LeMaire SA, Preventza O, de la Cruz KI, Cooley DA, Price MD, et al. Outcomes of 3309 thoracoabdominal aortic aneurysm repairs. J Thorac Cardiovasc Surg. 2016;151:1323-37.

2. Conrad MF, Crawford RS, Davison JK, Cambria RP. Thoracoabdominal aneurysm repair: a 20-year perspective. Ann Thorac Surg. 2007;83:S856-61; discussion S90-2.

3. Estrera AL, Sandhu HK, Charlton-Ouw KM, Afifi RO, Azizzadeh A, Miller CC III, et al. A quarter century of organ protection in open thoracoabdominal repair. Ann Surg. 2015;262:660-8.

4. Lombardi JV, Carpenter JP, Pochettino A, Sonnad SS, Bavaria JE. Thoracoabdominal aortic aneurysm repair after prior aortic surgery. $J$ Vasc Surg. 2003;38:1185-90.

5. Schlosser FJ, Mojibian H, Verhagen HJ, Moll FL, Muhs BE. Open thoracic or thoracoabdominal aortic aneurysm repair after previous abdominal aortic aneurysm surgery. J Vasc Surg. 2008;48:761-8.

6. Svensson LG, Crawford ES, Hess KR, Coselli JS, Safi HJ. Experience with 1509 patients undergoing thoracoabdominal aortic operations. J Vasc Surg. 1993;17: 357-68; discussion 368-70.

7. Chiesa R, Tshomba Y, Melissano G, Marone EM, Bertoglio L, Setacci F, et al. Hybrid approach to thoracoabdominal aortic aneurysms in patients with prior aortic surgery. J Vasc Surg. 2007;45:1128-35.

8. Schepens MA, Heijmen RH, Ranschaert W, Sonker U, Morshuis WJ. Thoracoabdominal aortic aneurysm repair: results of conventional open surgery. Eur J Vasc Endovasc Surg. 2009;37:640-5.

9. Etz CD, Zoli S, Mueller CS, Bodian CA, Di Luozzo G, Lazala R, et al. Staged repair significantly reduces paraplegia rate after extensive thoracoabdominal aortic aneurysm repair. J Thorac Cardiovasc Surg. 2010; 139:1464-72.

10. Zoli S, Etz CD, Roder F, Brenner RM, Bodian CA, Kleinman G, et al. Experimental two-stage simulated repair of extensive thoracoabdominal aneurysms reduces paraplegia risk. Ann Thorac Surg. 2010;90:722-9.

11. Etz CD, Zoli S, Kari FA, Mueller CS, Bodian CA, Di Luozzo G, et al. Redo lateral thoracotomy for reoperative descending and thoracoabdominal aortic repair: a consecutive series of 60 patients. Ann Thorac Surg. 2009;88:758-66; discussion 767 .

12. Kawaharada N, Morishita K, Fukada J, Watanabe T, Abe T. Surgical treatment of thoracoabdominal aortic aneurysm after repairs of descending thoracic or infrarenal abdominal aortic aneurysm. Eur J Cardiothorac Surg. 2001;20: $520-6$.

13. Kawaharada N, Morishita K, Fukada J, Hachiro Y, Takahashi K, Abe T. Thoracoabdominal aortic aneurysm repair through redo left-sided thoracotomy. Ann Thorac Surg. 2004;77:1304-8.

14. LeMaire SA, Carter SA, Volguina IV, Laux AT, Milewicz DM, Borsato GW, et al. Spectrum of aortic operations in 300 patients with confirmed or suspected Marfan syndrome. Ann Thorac Surg. 2006;81:2063-78; discussion 2078. 
15. Coselli JS, LeMaire SA, Buket S, Berzin E. Subsequent proximal aortic operations in 123 patients with previous infrarenal abdominal aortic aneurysm surgery. J Vasc Surg. 1995;22:59-67.

16. LeMaire SA, Price MD, Green SY, Zarda S, Coselli JS. Results of open thoracoabdominal aortic aneurysm repair. Ann Cardiothorac Surg. 2012;1:286-92.

17. Levey AS, Stevens LA, Schmid CH, Zhang YL, Castro AF III. Feldman HI, et al. A new equation to estimate glomerular filtration rate. Ann Intern Med. 2009;150: 604-12.

18. Coselli JS, de la Cruz KI, Preventza O, LeMaire SA, Weldon SA. Extent II thoracoabdominal aortic aneurysm repair: how I do it. Semin Thorac Cardiovasc Surg. 2016;28:221-37

19. LeMaire SA, Miller CC III, Conklin LD, Schmittling ZC, Koksoy C, Coselli JS. A new predictive model for adverse outcomes after elective thoracoabdominal aortic aneurysm repair. Ann Thorac Surg. 2001;71:1233-8.

20. Coselli JS, Poli de Figueiredo LF, LeMaire SA. Impact of previous thoracic aneurysm repair on thoracoabdominal aortic aneurysm management. Ann Thorac Surg. 1997;64:639-50.
21. Schepens MA, Kelder JC, Morshuis WJ, Heijmen RH, van Dongen EP, ter Beek HT. Long-term follow-up after thoracoabdominal aortic aneurysm repair. Ann Thorac Surg. 2007;83:S851-5; discussion S90-2.

22. Hiratzka LF, Bakris GL, Beckman JA, Bersin RM, Carr VF, Casey DE Jr, et al 2010 ACCF/AHA/AATS/ACR/ASA/SCA/SCAI/SIR/STS/SVM guidelines for the diagnosis and management of patients with thoracic aortic disease: a report of the American College of Cardiology Foundation/American Heart Association Task Force on Practice Guidelines, American Association for Thoracic Surgery, American College of Radiology, American Stroke Association, Society of Cardiovascular Anesthesiologists, Society for Cardiovascular Angiography and Interventions, Society of Interventional Radiology, Society of Thoracic Surgeons, and Society for Vascular Medicine. Circulation. 2010; 121:e266-369.

Key Words: aneurysm (aorta), aortic dissection, aortic operation, reoperation, thoracoabdominal, outcomes 
TABLE E1. Variables entered into multivariable logistic regression models (Tables 5 and 7)

Preoperative variables

Reoperative status

Male sex

Age, y

Symptomatic

Peripheral vascular disease

Connective tissue disorder

Marfan syndrome

Hypertension

Hyperlipidemia

Diabetes

Previous CABG

Previous MI

Coronary artery disease

Cerebrovascular disease

History of renal failure or dialysis

Chronic renal insufficiency

Pulmonary history

History of stroke

Current tobacco use

Rupture

Body mass index, initial serum creatinine level, estimated glomerular filtration rate, maximum distal diameter, and diameter $>7.5 \mathrm{~cm}$ had $P<.1$ in the univariate comparisons but were excluded because values were missing for approximately $10 \%$ of patients. TAAA, Thoracoabdominal aortic aneurysm; $C A B G$, coronary artery bypass grafting; $M I$, myocardial infarction.

\section{Intraoperative variables}

Urgent or emergent repair

Extraction of endograft

Reverse elephant trunk

Elephant trunk completion repair

Clamping proximal to left subclavian artery

Intercostal/lumbar artery reattachment

Splenectomy

Endarterectomy, stenting, or bypass

Extent II TAAA repair 